\title{
Use of non-invasive ventilation in acute respiratory failure due to SARS-CoV-2 pneumonia: typing of patients and choice of respiratory support, the role of internal medicine
}

\author{
Federico Lari, ${ }^{1}$ Stefania Guerrini, ${ }^{2}$ Fabrizio Giostra ${ }^{2}$ \\ ${ }^{1}$ Medicina Interna, AUSL Bologna; ${ }^{2}$ Medicina d'Urgenza e Pronto Soccorso, AOSP Bologna, Policlinico di S. Orsola, Italy
}

\begin{abstract}
The use of non-invasive ventilation (NIV) during de novo acute hypoxemic respiratory failure is not recommended by the guidelines because NIV does not improve the prognosis. With the advent of the new coronavirus, many cases of acute hypoxemic respiratory failure associated with the infection (severe acute respiratory infection) have been observed: data are missing regarding the use of NIV in this particular clinical condition, but a correct typing of patients based on different clinical, pathophysiological and radiological characteristics, could help in prognostic stratification and choice of respiratory support (invasive versus non-invasive). During NIV in these patients, particular attention is paid to the possibility of environmental dissemination of the virus, and consequently, adequate technical precautions are taken.
\end{abstract}

\section{Introduction}

The choice of respiratory support during acute respiratory failure (ARF) due to severe acute respiratory syndrome-coronavirus-2 (SARS-CoV-2) pneumonia is a topic widely debated in the last months: due to the lack of reliable data published in literature during this new pandemic, the Evidence-Based Medicine paradigm has been reversed and, when it comes to medical treatment, as well as to oxygenation/ventilation tech-

Correspondence: Federico Lari, Medicina Interna AUSL Bologna, via Enzo Palma 1, 40017 San Giovanni in Persiceto (BO), Italy.

E-mail: federico.lari@ausl.bologna.it

Key words: Acute respiratory failure; non-invasive ventilation; SARS-CoV-2.

Contributions: the authors contributed equally.

Conflict of interests: the authors declare no potential conflict of interests.

Ethics approval and consent to participate: not required.

Received for publication: 3 May 2020.

Revision received: 21 May 2020.

Accepted for publication: 22 May 2020.

This work is licensed under a Creative Commons Attribution NonCommercial 4.0 License (CC BY-NC 4.0).

${ }^{\circ}$ Copyright: the Author(s), 2020

Licensee PAGEPress, Italy

Italian Journal of Medicine 2020; 14:119-125

doi:10.4081/itjm.2020.1309 niques we rely on the experience gained in similar clinical conditions and on good clinical practice..$^{1-7}$ In the first weeks of pandemic, many patients were managed early with invasive ventilation as for other similar forms of ARF, such as acute adult respiratory distress syndrome (ARDS), and subsequently we verified that patients did not improve substantially in most of the cases. However, these patients often arrived in hospital in severe conditions after a first prolonged phase of untreated disease.

ARF typically occurs in the second week of illness in these patients. After an initial period of viral replication with fever and general nonspecific symptoms, a subsequent phase follows in which the excessive inflammatory response in some patients contributes to lung damage with diffuse alveolar impairment, inflammatory infiltrates, edema-exudate, endothelial damage (perhaps the main problem) with vascular thrombosis and disseminated intravascular coagulation. ${ }^{8}$ Among these, endothelial and vascular alterations seem to be decisive in the genesis of organ damage (also in other districts such as kidney, brain, and heart) and, as regards ARF, this would explain the inadequate response to mechanical ventilation especially to the invasive one that, if applied with high pressures, it could also worsen the respiratory failure. In a subsequent moment of the pandemic it was therefore understood that, perhaps, the disease had to be treated early trying to limit viral replication and containing the exaggerated inflammatory response and, as regards the choice of respiratory support in patients with ARF, we understood that it was fundamental a careful selection of patients to treat with invasive ventilation. At the same time, non-invasive strategies took on an increasingly important role. 


\section{Typing of patients with acute respiratory failure due to SARS-CoV-2 pneumonia}

Patient with respiratory failure secondary to SARS-CoV-2 pneumonia typically presents with hypoxemia, generally with respiratory alkalosis if ventilation is preserved, a reduction in the $\mathrm{PaO}_{2} / \mathrm{FiO}_{2}$ ratio, an increase in the arterial alveolar $\mathrm{O}_{2}$ gradient (the latter parameter is definitely more useful both for uncovering a gas exchange defect in patients with borderline $\mathrm{PaO}_{2} / \mathrm{FiO}_{2}$ values and for roughly quantifying its size, therefore, it should be used routinely). The mechanisms of hypoxemia are linked to alterations in the ventilation/perfusion ratio (primarily for vascular damage) with a variable share of shunt effect in the alveolar areas completely excluded from ventilation. ${ }^{9}$

The duration of respiratory failure is generally prolonged, whereby patients who need respiratory support are usually treated for a long period. It is crucial to know how to choose the appropriate respiratory support (non-invasive versus intubation) because the best way to minimize complications related to ventilation (invasive in particular) is to avoid unless absolutely necessary. And it has long been proven that the liberal versus conservative use of oxygen and ventilation has increased mortality in various clinical conditions. ${ }^{9}$ If in general we can claim to know the indications for intubation and invasive ventilation in other forms of ARF, this is not yet clarified for the severe acute respiratory infection (SARI) due to the new coronavirus: to traditional indicators that orientate towards intubation (such as lack of clinical and gas exchange improvement during NIV with persistence of respiratory distress, hypoxemia and acidosis, hemodynamic instability, cardiac or respiratory arrest, airway obstruction, abundant and unmanageable secretions, worsening of neurological status, need to protect the airways $)^{1,2,3,6,7}$ for SARS-CoV-2, the literature is evaluating other prognostic indicators that direct towards the choice of treating the patient in an invasive or non-invasive way (respiratory work evaluated for example with esophageal manometry, quantification of ventilated areas of the lung at computed tomography (CT) scan). ${ }^{10,11}$

Clinically the patient with ARF due to SARS-CoV2 pneumonia, more often a middle-advanced aged man, may not be clearly dyspneic: in general, the respiratory rate is not excessively high, the breathing is deep, with high tidal volumes, there are no signs of respiratory distress or use of the accessory respiratory muscles (for example, the absence of phasic contraction of the sternocleidomastoid muscle ${ }^{9}$ ) and the patient can easily tolerate compromised oxygenation values. The organ damage during hypoxemia depends not only on the absolute values of $\mathrm{PaO}_{2}$ but also on cardiac output and tissue oxygen extraction which usually increase in a compensatory way during hypoxemia, making evident the organ damage for $\mathrm{PaO}_{2}$ values lower than $40 \mathrm{mmHg} .{ }^{9}$ Radiological findings (high-resolution CT scan, HRCT) are generally a widespread interstitial thickening, ground-glass areas (preferably peri-septic at the beginning), more frequently a subpleural consolidation, and sometimes, minimal pleural effusion. The ultrasound shows a mixed pattern with bilateral non-symmetrical B lines, and white lung areas, irregularities of the pleural line, subpleural thickenings, and pleural effusion will also be highly appreciable. According to the indications of literature, ${ }^{12-14}$ a patient of this type can be classified as phenotype $L$, in which lung compliance is typical, and this could explain the absence of respiratory distress by differentiating it from other forms of ARDS.

The second phenotype described in the literature ${ }^{12-14}$ is phenotype $H$ in which lung compliance is reduced, and it represents approximately $20 \%$ of patients with SARS-CoV-2 pneumonia and respiratory failure. This phenotype can be the evolution of the disease (from phenotype $\mathrm{L}$ to phenotype $\mathrm{H}$ ) due to the worsening of lung damage which can also be caused by respiratory distress, that, in these cases is usually present and severe (patient self-inflicted lung injury, due to the significant negative values of intrathoracic pressure during inspiration measurable with esophageal manometry) or even caused by high pressures in the airways during mechanical ventilation (ventilatoryinduced lung injury). In these patients, the HRCT scan shows more severe findings with worsening of edema, large consolidation areas, severe reduction of the pulmonary ventilation due to alveolar collapse, and shunt effect. This condition is very similar to other forms of ARDS and meets the criteria of Berlin Definition. ${ }^{15}$

The distinction between the two phenotypes described above, characterized by different pathophysiological mechanisms (Figure 1), is essential for the overall management of the patient and also for the choice of respiratory support., ${ }^{9} 12-14$ Patient $\mathrm{L}$ is probably a patient at an earlier stage of his respiratory disease, less severe, and should be managed conservatively, non-invasively, avoiding intubation even for compromised oxygenation levels with the aim of giving time to disease and medical treatment to take the natural course. Its management would, therefore, deviate from the indications proposed by the guidelines on ARDS, which indicate early intubation and marginal role of NIV (Figure 2). The most severe $\mathrm{H}$ phenotype, on the other hand, looks much more like other forms of ARDS and, therefore, it should be managed as such, preferably invasively without delaying intubation. ${ }^{16}$ It is true, however, that in many cases, even if intubation would be indicated based on clinical, radiological and gas-analytical criteria, in practice 

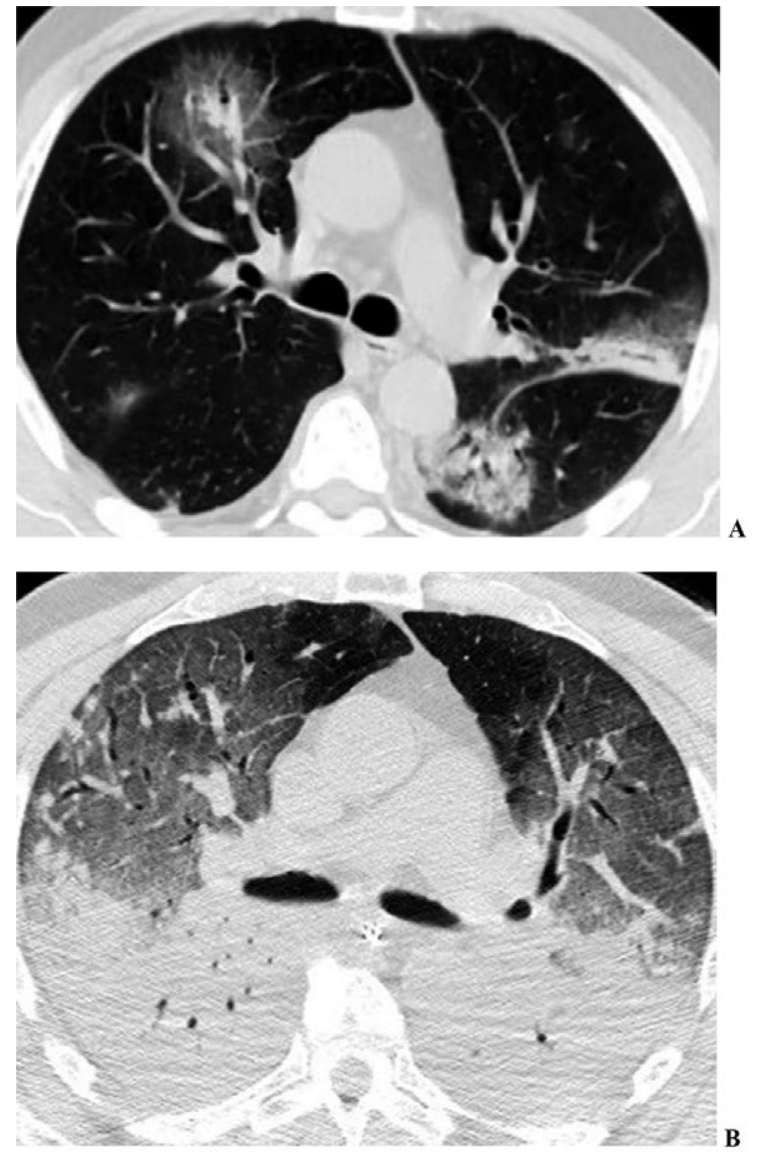

Figure 1. Phenotypes of lung damage in SARS-CoV-2 pneumonia. A) Phenotype L: -normal compliance: the amount of gas in the lungs is almost regular or slightly altered. - impairment of ventilation/perfusion ratio as the primary mechanism of hypoxemia. - Lung weight only slightly increased: only small areas of ground glass and small consolidations are present. - Low recruitability: the amount of non-aerated tissue is low (use of low positive end-expiratory pressures, PEEP). - absent or mild respiratory distress evaluable with the recruitment of accessory muscles (e.g. absence of phasic contraction of the sternocleidomastoid muscle), respiratory rate alone is not an expression of increased work of breathing. - acceptable response to oxygen therapy / NIV; B) Phenotype H: - low compliance: the amount of gas in the lungs is reduced due to the presence of an abundant amount of edema. - shunt effect as the main mechanism of hypoxemia, the cardiac output perfuses large areas that are not ventilated for edema. - Lung weight significantly increased due to the presence of large areas of consolidation and ground glass as in other forms of adult respiratory distress syndrome. - High recruitability: the presence of large areas of nonaerated parenchyma theoretically conditions a high possibility of alveolar recruitment (use of high PEEP and prone position). - respiratory distress: evaluable with the recruitment of accessory muscles (e.g., presence of phasic contraction of the sternocleidomastoid muscle) or with esophageal manometry, which shows wide variations in intrapleural pressure. - inadequate response to oxygen therapy / NIV. Modified from L. Gattinoni et al., 2020: https://doi.org/10.1007/s00134-020-06033-2. it is not feasible because the patient is elderly, suffering from comorbidities and critical chronic diseases, thus remaining only suitable for non-invasive management outside intensive care units.

\section{Choosing respiratory support}

We now provide some practical indications for the choice of non-invasive respiratory support in patients with L phenotype, which are the majority of SARS$\mathrm{CoV}-2$ patients with respiratory failure and are usually managed outside intensive care units. ${ }^{5-7}$

The first approach to manage hypoxemia should be through standard oxygen therapy, such as the Venturi mask that guarantees high flows as well as precise and high $\mathrm{FiO}_{2}$. It is appropriate to increase $\mathrm{FiO}_{2}$ progressively if hypoxemia is not corrected and take care to maintain a surgical mask over the Venturi mask to limit the viral dispersion. Nasal prongs are less effective in acute patients in terms of flow and $\mathrm{FiO}_{2}$ and increase the environmental dispersion so they would not be indicated. If the Venturi mask is not sufficient to guarantee adequate oxygenation, it is possible to switch to the reservoir mask that provides high $\mathrm{FiO}_{2}$ in patients without high inspiratory flow (slightly dyspnoic) or to the high flow nasal cannulas with a heated and humidified mixture (HFNC): although this system is also burdened by the risk of viral dispersion (and therefore can be used preferentially in environments with negative pressure), it is effective because it guarantees high flows, precise $\mathrm{FiO}_{2}$, and excellent patient comfort. Also in this case, the surgical mask correctly positioned above the cannulas is a must. If oxygen therapy is ineffective, the next step in terms of therapeutic aggression is non-invasive ventilation (NIV) in continuous positive airways pressure CPAP mode or double pressure level (Table 1) which should also be practiced, to limit the exposure of operators, in environments with negative pressure and especially in critical areas with adequate monitoring, expert staff, rapid access to intubation and intensive care units in case of worsening. The application of CPAP reduces the negative intrathoracic pressure during inspiration, improving the ventilation/perfusion ratio recruiting alveolar units with positive effects on oxygenation. In this particular clinical condition, we must pay attention to the patient's hemodynamic status because if we use too high positive end-expiratory pressures (PEEP), besides the risk of worsening lung damage due to overdistention of alveoli not affected by exudate, we could compromise the venous return and cardiac output and, therefore, the oxygen delivery to tissues. In general, pressure support during inspiration is not necessary in these patients because, in most cases, they maintain normal/high tidal volumes and proper lung ventilation, in the absence of pre-existing ventilation 
problems. Indeed it is important not to increase the tidal volume, which could worsen lung damage.

CPAP can be delivered with different systems: ${ }^{5-7}$

- Venturi likeflow generators: they can be integrated into the system (disposable) or external with the machine body. They are certainly the best performing systems for CPAP because they use high flows capable of satisfying the inspiratory needs of even the most dyspnoic patients; however they are probably the most burdened systems by the viral environmental dispersion because the high excess flow is generally eliminated in the environment through the PEEP valve. It is therefore advisable, in this situation, to use them as a second choice and combine at least 2 antibacterial/antiviral filters, one positioned between the machine body and the circuit (or at the entrance of the window that recalls ambient air) and a second immediately before the PEEP valve (for example between mask or helmet and valve).

- Ventilators: generally, all NIV ventilators with turbine technology offer the possibility of a CPAP mode. Because they are technologically different from Venturi flow generators, they generally use lower flows that adapt in real time to patient's inspiratory needs. For this reason, they are certainly safer as regards the viral dispersion but less performing in keeping the pressures constant during inspiration if the patient has high peak flow. With a dual limb circuit, the environmental dispersion is probably less because the expiratory line conveys the exhaled into the machine with further filtering: however it is advisable to use antibacterial and antiviral filters at the entrance of the inspiratory line, at the exit of the expiratory line, and between the mask or helmet and the circuit. Single limb ventilators with an expiratory valve or intentional leaks that eliminate the expired gases in the external environment are probably more risky for the viral dispersion: in this case, it is advisable to use two antibacterial and antiviral filters, one at the circuit input (between ventilator and tube), the second between mask or helmet and expiratory valve or intentional leak (usually placed at the end of the tube, close to the patient interface).

The choice of interface is crucial ${ }^{5-7}$, as this affects

Table 1. Practical tips for the choice of respiratory support in acute respiratory failure due to SARS-CoV-2 pneumonia.

\begin{tabular}{lllcc}
\hline $\mathbf{S p O}_{\mathbf{2}}$ \%* & & RR & in & Switch to \\
\hline$<95$ & and & $>20$ & AA & Ventimask o Reservoir \\
\hline$<92$ & and/or & $>20$ & Ventimask or Reservoir & HFNC/CPAP/Bilevel \\
\hline$<92$ & and/or & $>25$ & HFNC & CPAP/Bilevel \\
\hline$<92$ & and/or & $>25$ & CPAP/Bilevel & Consider intubation \\
\hline
\end{tabular}

*Target $\mathrm{SpO}_{2}$ 92-96\%, 88-92\% (if chronic ventilatory failure is present). $\mathrm{SpO}_{2}$, partial saturation of oxygen; RR, respiratory rate; $\mathrm{AA}$, ambient air; HFNC, high flow nasal cannula; CPAP, continuous positive airways pressure.

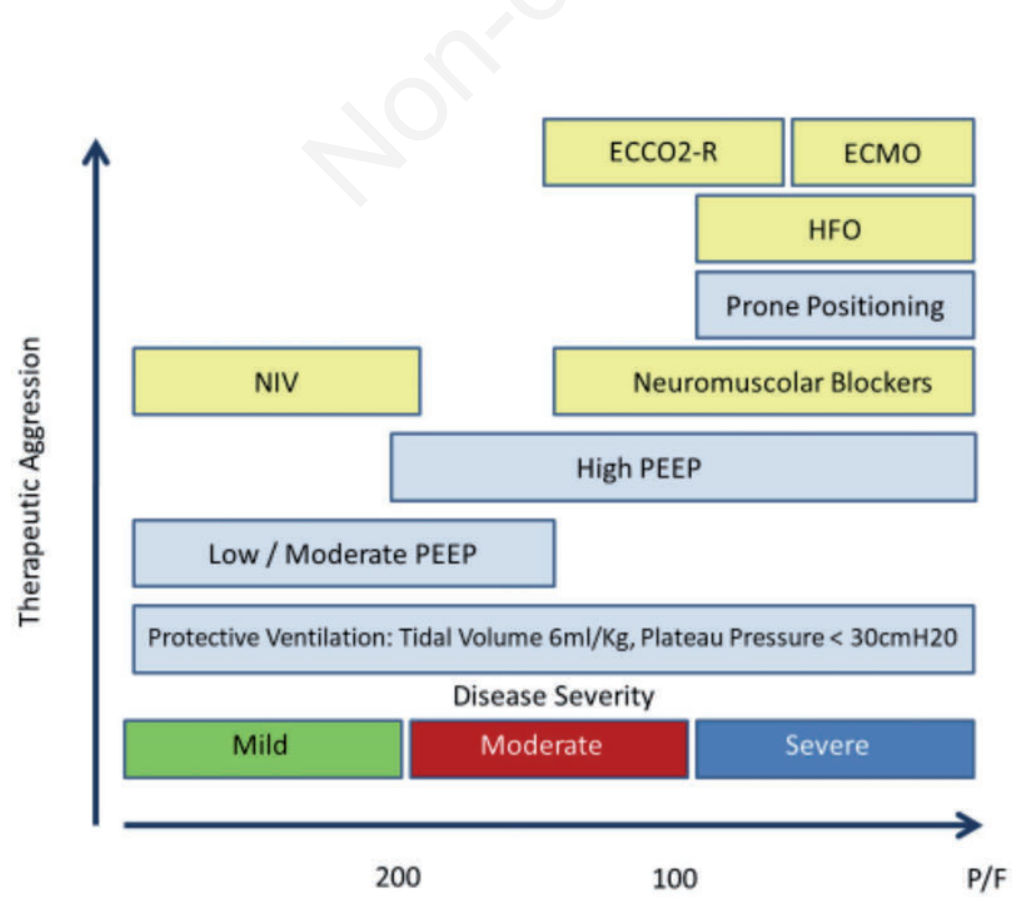

Figure 2. Respiratory support in adult respiratory distress syndrome. $\mathrm{P} / \mathrm{F}$, $\mathrm{PaO}_{2} / \mathrm{FiO}_{2}$ (obtained in continuous positive airways pressure). NIV, non-invasive ventilation; PEEP, positive end-expiratory pressure; HFO, high-frequency oscillation; ECMO, extracorporeal membrane oxygenation; ECCO2-R, extracorporeal $\mathrm{CO}_{2}$ removal. 
both the effectiveness of treatment and the risk of environmental viral dissemination. In general, it is advisable to use non-vented masks, i.e. without intentional leaks (which must be present on the circuit if a single limb ventilator is used). The face mask (oronasal) remains the most suitable in case of emergency, the best in terms of efficacy (constant pressures and $\mathrm{FiO}_{2}$ ), but it can expose to the risk of leaks and, therefore, of environmental dissemination as well as the total face mask. From this point of view, the helmet seems to offer some more guarantees even if not all turbine ventilators provide adequate flows to avoid $\mathrm{CO}_{2}$ rebreathing with the consequent risk of hypercapnia. Furthermore, if used in bilevel pressure mode, the helmet could generate problems related to the activation of the triggers and the monitoring of the current volume with some ventilators. The helmet also offers the advantage of better comfort for the patient undergoing prolonged treatments, as it usually happens in this clinical condition, it allows the patient to drink, and the medical staff to introduce probes through special openings, on the other hand, the noise perceived by the patient can be annoying (but earplugs can be used), it requires at least 2 operators and a few minutes to be positioned correctly, furthermore, it has a higher cost than the other interfaces. The moment when the helmet is removed can be critical for the environmental dispersion of the virus; this procedure must be done with caution by the staff placing themselves if possible behind the patient. In any case, before using the helmet with a turbine ventilator, it is important to contact the supplier/manufacturer of the machine and consult the instructions manual to check its compatibility. It may be advisable to start with a helmet in the first hours/days of treatment then, at the moment of weaning, to consider the possibility of switching to facial/total face masks. In this phase, it is fundamental to avoid long pauses from ventilation to prevent derecruitment, and to consider at any time the elective adaptation of each single patient to the different interfaces. Moreover, it is crucial to guarantee a clean skin, the use of protective barriers, and the rotation of different interfaces to prevent pressure injuries. In the literature, there are few data on the effectiveness of pronation (even partial) during NIV in improving oxygenation parameters; however, it is an option to consider before sanctioning the failure of NIV.

In Table 2, we report some practical indications regarding the use of NIV in patients with ARF due to SARS-CoV-2 pneumonia.

\section{Monitoring}

Like all patients undergoing oxygen therapy, and even more in those whose treatment involves the application of positive pressures, a continuous, simple, and non-invasive monitoring is essential. ${ }^{5-7}$ Among the parameters to be assessed, $\mathrm{SpO}_{2}$ is certainly fundamental even if, especially in the early stages, it can be

Table 2. Practical indication for the use of non-invasive ventilation in acute respiratory failure due to SARS-CoV-2 pneumonia.

- First choice: dual limb ventilator with helmet (if compatible = adequate flows to avoid rebreathing) and 3 hygroscopic antibacterial and antiviral filters (at the entrance of the inspiratory line, at the exit of the expiratory line and between the helmet and the expiratory line / Y circuit, to be replaced every $12 / 24 \mathrm{~h}$ )

- Second choice: single limb ventilator with expiratory valve / intentional leaks and helmet, 2 antibacterial and antiviral hygroscopic filters, one at the circuit inlet (between fan and tube), the second between interface and expiratory valve / intentional leak

- Third choice: Venturi flow generator with balloon reservoir and helmet, 2 antibacterial and antiviral hygroscopic filters, one positioned between the machine body and circuit or at the inlet of the window that recalls ambient air and a second between interface and PEEP valve

- Second choice: non-vented face/total face masks

- Mode: CPAP / PEEP 8-10 max $12 \mathrm{~cm} \mathrm{H}_{2} \mathrm{O}$

- $\mathrm{FiO}_{2}$ adequate to maintain $\mathrm{SpO}_{2} 92-94 \%$, do not exceed $\mathrm{SpO}_{2} 96-98 \%$

- If a bilevel or pressure support mode is used: Pressure Support set at $6 \mathrm{~cm} \mathrm{H} \mathrm{H}_{2} \mathrm{O}$ and not more than $15 \mathrm{~cm} \mathrm{H}_{2} \mathrm{O}$ (avoid high tidal volumes, target 4-6 $\mathrm{mL} / \mathrm{kg}$ predicted body weight), rise time short, inspiratory trigger set to avoid auto-triggering or ineffective effort, flow cycling set at $20 \%$ of inspiratory peak flow (if present COPD with flow limitation, set at $40 \%$ of the peak), maximum inspiratory time $1.25 \mathrm{~s}$, back up RR 10 acts/min with an inspiratory time of 1 second

- Clinical and gas analytic control 2 hours from the beginning of NIV, if no improvement (RR, respiratory pattern, $\mathrm{SpO}_{2}, \mathrm{PaO}_{2} / \mathrm{FiO}_{2}$, neurological score according to Kelly) consider intubation and invasive ventilation

- Use of scores during monitoring such as MEWS or ROX index $\left(\mathrm{SpO}_{2} / \mathrm{FiO}_{2} /\right.$ respiratory rate): the latter is a good predictor of HFNC treatment failure in patients with pneumonia. Reference values in this context are: ROX $>4.88$ at 2-6-12 $\mathrm{h}$ from the beginning of treatment $=$ low risk of failure / intubation, $\operatorname{ROX}<2.85$ at 2 hours, $<3.47$ at 6 hours, $<3.85$ at 12 hours $=$ high risk of failure / intubation. Its use is possible for the monitoring of non-invasive treatment in patients with ARF due to SARS-CoV-2 pneumonia

PEEP, positive end-expiratory pressures; CPAP, continuous positive airways pressure; COPD, chronic obstructive pulmonary disease; NIV, non-invasive ventilation; RR, respiratory rate; MEWS, Modified Early Warning Score; ROX, oxygen saturation as measured by pulse oximetry/ $\mathrm{FiO}_{2}$ to respiratory rate; HFNC, high flow nasal cannula; ARF, acute respiratory failure. 
falsely reassuring. This is due to the fact the the patient with phenotype L, hyperventilating, corrects the hypoxemia quite well and determines a shift to the left of the hemoglobin dissociation curve caused by hypocapnia and respiratory alkalosis. A critical indicator is the respiratory rate (RR) even if, like $\mathrm{SpO}_{2}$, at the beginning of the disease, it can show values that deviate little from the norm despite severe hypoxemia. Heart rate and blood pressure represent other two values to be measured regularly. Fluid balance must be absolutely measured in order to avoid its positivity (a situation that would worsen the alveolar edema). Arterial blood gas analysis represents, as in other forms of respiratory failure, the main examination. However, if the check performed at time zero is essential, too close checks should be avoided given the long duration of the respiratory support. Check-ups should be performed at least every $24 \mathrm{~h}$ unless in the presence of major changes in patient's clinical condition. In this form of hypoxemic and non-hypercapnic respiratory failure, the frequency of blood gases could be reduced by monitoring the ROX index..$^{17}$ The advantage of this index is that it is totally non-invasive and easy to apply. It is calculated using the following formula: $\left(\mathrm{SpO}_{2} / \mathrm{FiO}_{2}\right) / \mathrm{RR}$. Although it has been mainly analyzed in a study on pneumonia treated with high flows with nasal cannulas (where it was shown that values below 4.8 had a negative prognostic significance), this index can certainly provide a response trend to therapy and alert us when it drops below 8 . Lastly, thoracic ultrasound is undoubtedly very useful in monitoring the treatment.

\section{Conclusions}

In general, NIV in acute hypoxemic respiratory failure (de novo ARF) such as SARI, pneumonia, other pandemics due to respiratory viruses, and ARDS, is not indicated by the Guidelines because it is not effective in reducing mortality and the need for intubation. For SARS-CoV-2, however, the role of NIV is still unclear and must be defined.

In patients with SARI COVID-19 related and phenotype L without severe respiratory distress, HFNC and NIV could have a role in improving dyspnea and oxygenation when traditional oxygen therapy is not effective; in these patients, we should try to avoid intubation in order to prevent a hasty procedure potentially causing complications and worsening of lung damage.

The most severe patients with respiratory distress and phenotype $\mathrm{H}$ should instead be managed invasively according to the ARDS guidelines; therefore, the role of NIV is marginal, and intubation should not be delayed.

It is important to ventilate gently with low pres- sures (PEEP 8-10 $\mathrm{cm} \mathrm{H}_{2} \mathrm{O}$ ) also in NIV patients with phenotype L.

If possible, use systems, interfaces, and filters that limit the environmental dispersion of the virus as much as possible.

NIV and CPAP would preferably be used in protected environments (critical care areas) with negative pressure and experienced staff.

Finally, it is crucial to consider weaning the patient from mechanical ventilation, even from NIV, if respiratory distress is not present in oxygen therapy (preferably at high flows).

\section{References}

1. BTS guidelines. Non-invasive ventilation in acute respiratory failure. Thorax 2002;57:192-211.

2. Davidson AC, Banham S, Elliott M, et al. BTS/ICS guideline for the ventilatory management of acute hypercapnic respiratory failure in adults. Thorax 2016;71:ii1-ii35.

3. Rochwerg B, Brochard L, Elliott M, et al. Official ERS/ATS clinical practice guidelines: noninvasive ventilation for acute respiratory failure. Eur Respir J 2017;50:1-20.

4. Keenan SP, Sinuff T, Burns KEA, et al. Clinical practice guidelines for the use of noninvasive positive-pressure ventilation and noninvasive continuous positive airway pressure in the acute care setting. CMAJ 2011;183: E195-E214.

5. Navalesi P, Campanini M, Lari F, et al. La ventilazione non invasiva in medicina interna. Monografia. Quad Ital J Med 2015;3(1):391-498.

6. Lari F, Giostra F, Bragagni G, et al. La ventilazione meccanica non invasiva nell'insufficienza respiratoria acuta: stato dell'arte (Parte I). Ital J Med 2009;3:201-11.

7. Lari F, Giostra F, Bragagni G, et al. La ventilazione meccanica non invasiva nell'insufficienza respiratoria acuta: stato dell'arte (Parte II). Ital J Med 2010;4:6-15.

8. Alhazzani W, Hylander Møller M, Arabi YM. surviving sepsis campaign: guidelines on the management of critically ill adults with coronavirus disease 2019 (COVID19). Intensive Care Med 2020 [Epub ahead of print].

9. Tobin MJ. Basing respiratory management of coronavirus on physiological principles. Am J Respir Crit Care Med 2020 [Epub ahead of print].

10. Colombi D, Bodini FC, Petrini M, et al. Well-aerated Lung on Admitting Chest CT to Predict Adverse Outcome in COVID-19 Pneumonia. Radiology 2020 [In press].

11. Tonelli R, Fantini R, Tabbì L, et al. Inspiratory effort assessment by esophageal manometry early predicts noninvasive ventilation outcome in de novo respiratory failure: a pilot study. Am J Respir Critical Care Med 2020 [In press].

12. Gattinoni L, Chiumello D, Rossi S. COVID-19 pneumonia: ARDS or not? Critical Care 2020;24:154.

13. Gattinoni L. Chiumiello D, Caironi P, et al. COVID-19 pneumonia: different respiratory treatments for different phenotypes? Intensive Care Med 2020 [In press].

14. Marini J, Gattinoni L. Management of COVID-19 res- 
piratory distress. JAMA Insights 2020 [Epub ahead of print].

15. The ARDS Definition Task Force. Acute respiratory distress syndrome: The Berlin Definition. JAMA 2012;307:2526-33.

16. Papazian L, Aubron C, Brochard L, et al. Management of acute respiratory distress syndrome. Ann Intensive Care 2019;9:69.

17. Roca O, Caralt B, Messika J, et al. An index combining respiratory rate and oxygenation to predict outcome of nasal high-flow therapy. Am J Respir Crit Care Med 2019;199:1368-76. 